\title{
Hyperechoic Appearance of Malignant Phyllodes Tumor with Liposarcomatous Differentiation: A Case Report with Pathologic Correlation
}

\author{
Seung Min Kang ${ }^{1}$, Shin Young Kim ${ }^{1,{ }^{*}}$, Sun $\mathrm{Huh}^{1}$, Si Hyong Jang ${ }^{2}$ and Jong Eun Lee ${ }^{3}$ \\ ${ }^{1}$ Department of Radiology, Cheonan Hospital, Soonchunhyang University College of Medicine, Cheonan, Korea \\ ${ }^{2}$ Department of Pathology, Cheonan Hospital, Soonchunhyang University College of Medicine, Cheonan, Korea \\ ${ }^{3}$ Department of Surgery, Cheonan Hospital, Soonchunhyang University College of Medicine, Cheonan, Korea \\ "Corresponding author: Department of Radiology, Cheonan Hospital, Soonchunhyang University College of Medicine, 31 Soonchunhyang 6-gil, Dongnam-gu, \\ Chungcheongnam-do, Cheonan, Korea. Tel: +82-415703515, Fax:+82-415799026, Email: c87093@schmc.ac.kr \\ Received 2018 December 11; Revised 2019 March 04; Accepted 2019 May 20.
}

\begin{abstract}
A malignant phyllodes tumor is a rare disease of the breast that usually differentiates into fibrosarcoma, with liposarcomatous differentiation rarely being reported. In general, malignant phyllodes tumors appear as hypoechoic lesions on ultrasonography examination, and have rarely been reported as hyperechoic tumors. Here, we present an extremely rare case of a 62-year-old woman with a palpable mass that was diagnosed as a malignant phyllodes tumor with liposarcomatous differentiation, seen as an unusual hyperechoic mass on ultrasonography. She underwent resection and biopsy, and there was no evidence of tumor recurrence on follow-up examinations. Radiological explanations of this case are presented in detail, and the possible associations between hyperechoic appearance and liposarcomatous differentiation are described. Through our case report, we can identify several potential rare features of various and unpredictable phyllodes tumors.
\end{abstract}

Keywords: Breast, Phyllodes Tumor, Malignant Phyllodes Tumor, Mammography, Ultrasonography

\section{Introduction}

A phyllodes tumor is an uncommon breast tumor with a large, rapidly growing tendency (1). Phyllodes tumors can be benign, borderline, or malignant, and the prevalence of malignant tumors is varied but rare $(2,3)$. A malignant phyllodes tumor usually shows fibrosarcomatous differentiation and rarely shows other heterologous sarcomatous elements (4). On ultrasonography (USG), most of these tumors exhibited lobulated contours, smooth margins, hypoechoic echotexture, with posterior acoustic enhancement. Malignancy may be suspected if the tumor contains cystic space, or if it is with an irregular shape or non-circumscribed margin (5-7).

In the literature, malignant phyllodes tumors with hyperechoic appearance on USG have rarely been reported, and only one case of malignant phyllodes tumor showing hyperechoic appearance has been reported. That case showed liposarcomoatous differentiation, the same as in as our case (8). In this case report, we report a case of malignant phyllodes tumor with liposarcomatous differentiation observed as a predominantly hyperechoic mass with cystic portions on USG. Our case report includes serial mammography, USG images including Doppler USG, and microscope slides with pathologic explanations, compared to the previous case report which presented only one USG image. In our case report, detailed radiologic findings of malignant phyllodes tumors seen as hyperechoic on USG have been identified, and their relationship with liposarcomatous differentiation have been considered.

\section{Case Presentation}

A 62-year-old female patient visited our clinic with a palpable mass in her right breast. She had first noticed this tumor six months before her visit. The mass was a $4 \mathrm{~cm}$ sized firm and fixed mass in the upper outer quadrant of her right breast. There was no familial or psychosocial history related to breast disease.

Five years prior, she had visited an outside hospital with similar symptoms. On mammography in that visit, the mass was observed as a $2.5 \mathrm{~cm}$ sized oval circumscribed equal density mass with a radiolucent halo at the mid 
outer portion of her right breast (Figure 1). Excision and biopsy were performed in our hospital, because the mass was palpable and showed a relatively fast-growing tendency. The mass was diagnosed as a borderline phyllodes tumor, and the resection margins were positive for the tumor cells.

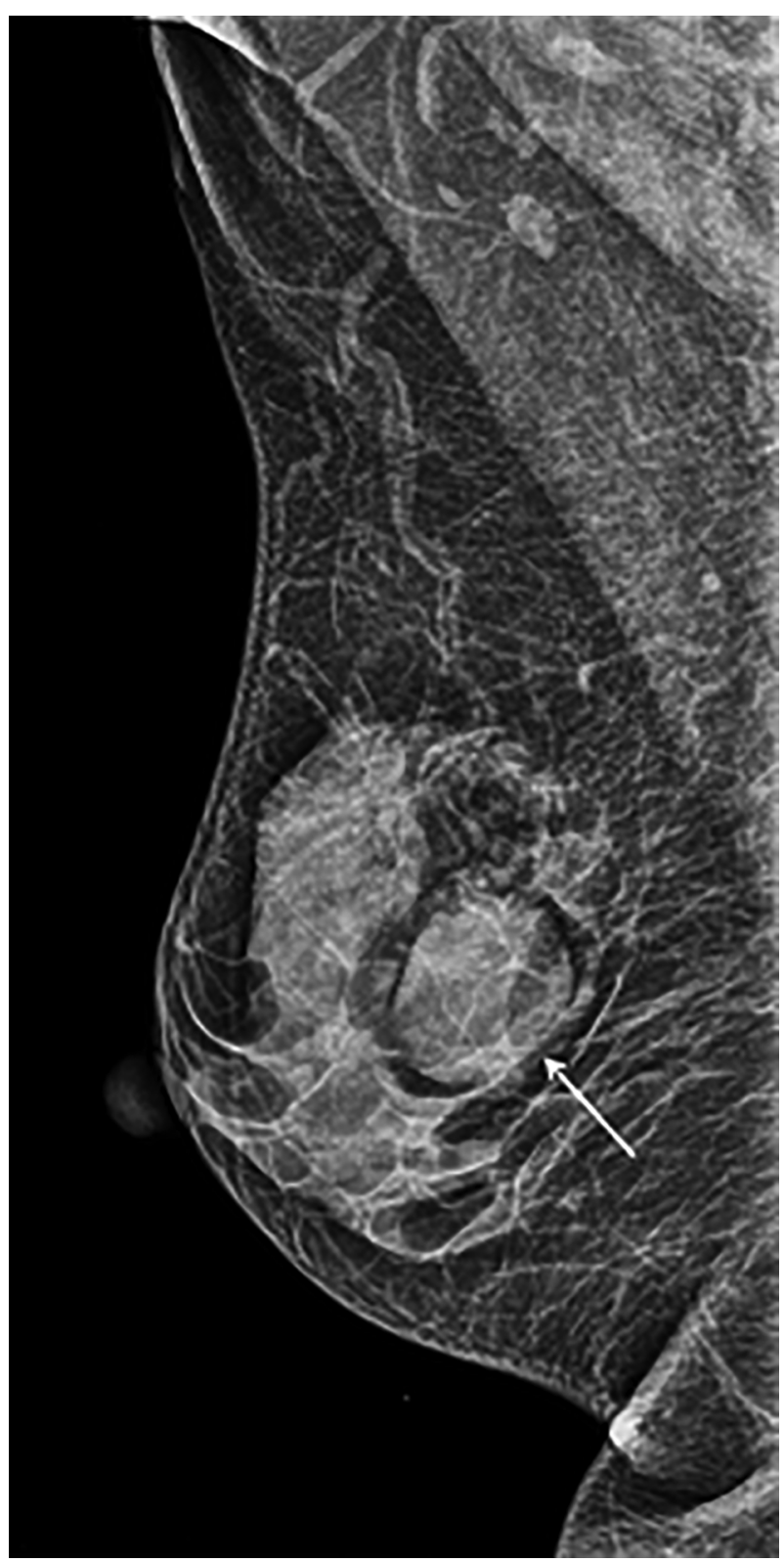

Figure 1. A 57-year-old female patient with a palpable mass in her right breast (five years ago). Mammography with mediolateral oblique (MLO) view of the right breast in a 57-year-old woman with a palpable mass. It was an approximately $2.5 \mathrm{~cm}$ sized oval circumscribed equal density mass (arrow) with a radiolucent halo at the midouter portion of the right breast.
The patient had taken serial follow-up mammographies at the outside hospital before returning to our hospital (Figure 2). On mammography taken two years prior, a newly developed mass was found in her right breast (Figure 2A). On mammography taken six months prior, the mass was observed to be larger, shown as a $3 \mathrm{~cm}$ sized oval indistinct equal density mass, with heterogeneous internal fat components (Figure $2 \mathrm{~B}$ ). The tumor then grew larger, to $4 \mathrm{~cm}$ in size, with more dense and lobulated morphology, so she returned to our hospital (Figure 2C).

On USG, the mass was an oval indistinct heterogeneous hyperechoic mass with cystic portions, and color Doppler ultrasound showed minimal internal vascularity (Figure 3). Since the margins were indistinct and the size rapidly increased, the likelihood of malignancy was considered high and subsequently classified as breast imaging-reporting and data system (BIRADS) category 4c. Core needle biopsy was performed for both hyperechoic and cystic portions, and the mass was diagnosed as a fibroepithelial lesion with atypical mesenchymal cell proliferation.

Excision and biopsy were subsequently performed on the mass because the mass was suspected to be malignant. Gross examination showed an ill-demarcated gray-white rubbery mass, measuring $4.0 \times 2.4 \times 1.7 \mathrm{~cm}$. Upon microscopic examination, the tumor showed a leaf-like growth pattern and hypercellular stroma (Figure 4A). The stroma consisted of severe pleomorphic polygonal to spindle cells with lipoblasts (Figure 4B and C). Finally, the mass was diagnosed as a malignant phyllodes tumor with liposarcomatous differentiation.

The surgical resection margins were negative for tumor cells, and the patient returned to our hospital 6 months after surgery. She underwent follow-up evaluation including mammography, breast USG, computed tomography of chest with magnetic resolution image of the breast, and there was no evidence of local recurrence or distant metastasis. Because the mass was completely removed and there was no evidence of recurrence, we decided to do an ultrasound every 6 months without any other medical treatment.

\section{Discussion}

Phyllodes tumor is named after a leaf-like configuration of an overgrown mesenchymal structure, and is a rare disease that occurs in less than $1 \%$ of all breast tumors (1). The phyllodes tumor is usually benign, but it may be borderline or malignant, and the percentage of malignant tumors reported in the literature varies from $8 \%$ to $45 \%(2,3)$. Most of the malignant phyllodes tumors show fibrosarcomatous differentiation, and other sarcomatous elements 

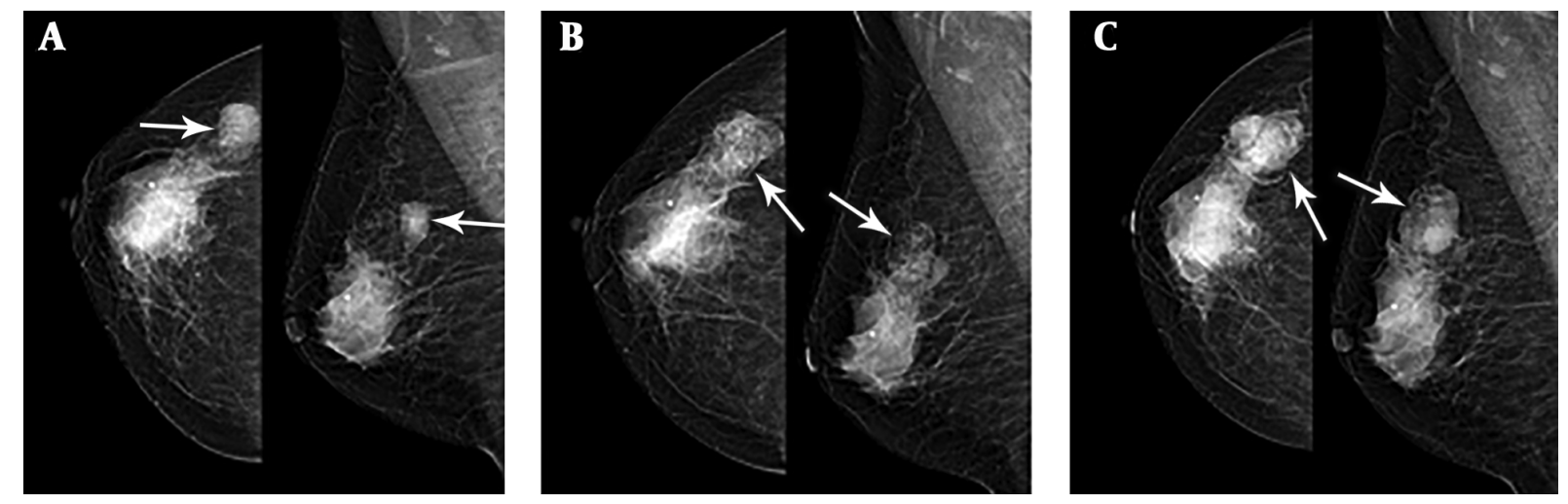

Figure 2. Serial mammography with craniocaudal (CC) and mediolateral oblique (MLO) view of the right breast. Two years (A), six months (B), and one day (C) before she returned to our hospital. Newly developed $2 \mathrm{~cm}$ sized round circumscribed equal density mass (arrows) in the upper outer quadrant of the right breast (A). The size increased to about $3 \mathrm{~cm}$, the margin became indistinct, density increased, and a new heterogeneous fat component was observed (arrows) (B). The size increased to approximately $4 \mathrm{~cm}$, and the density increased (arrows) (C).
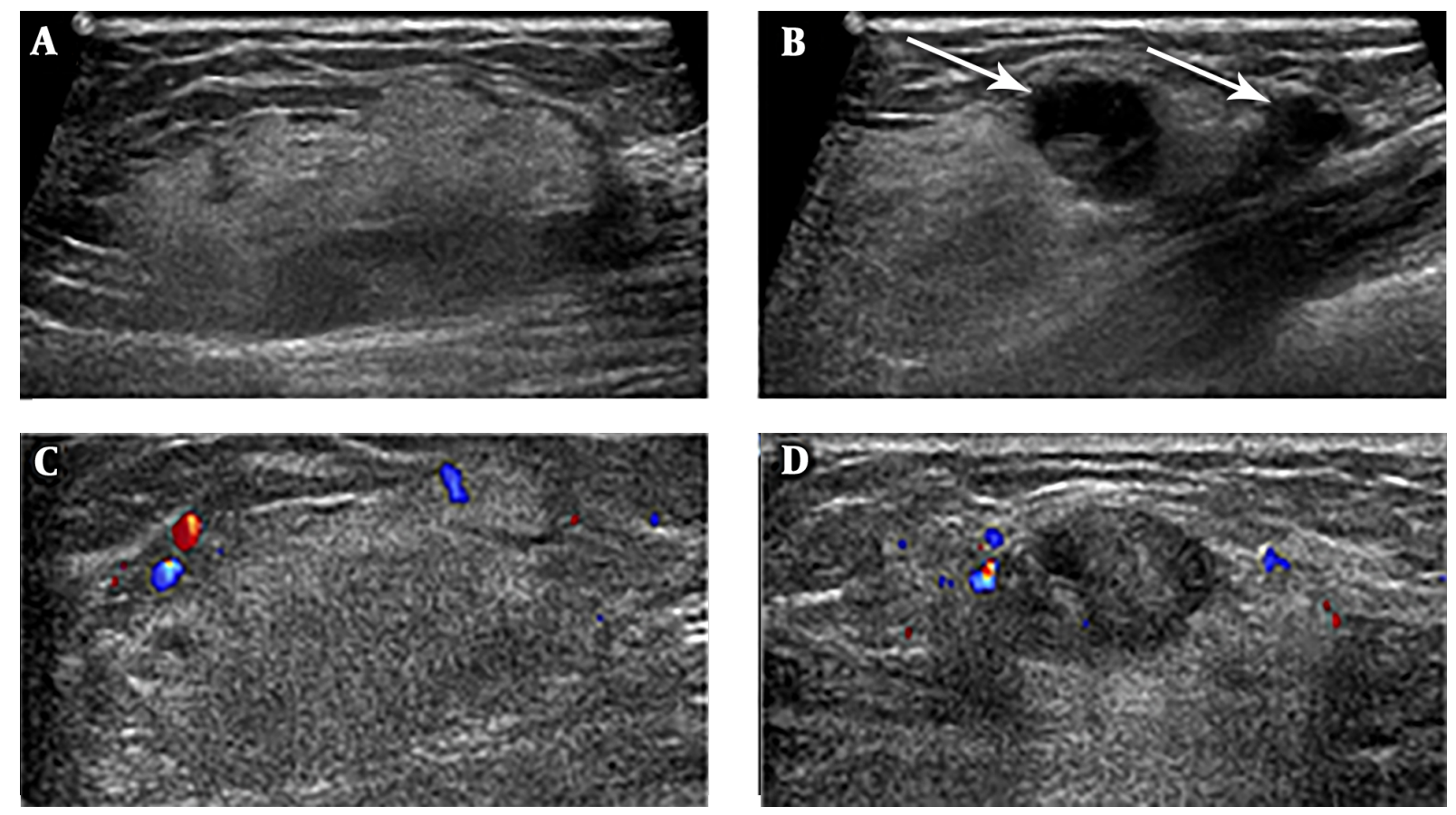

Figure 3. Ultrasonography (USG) of right breast with a recurred palpable mass five years after first visit (current age: 62 years). It was an approximately $4 \mathrm{~cm}$ sized oval indistinct heterogeneous hyperechoic mass (A), with cystic portions (arrows) (B), and color Doppler USG showed minimal internal vascularity (C, D).

are rarely reported. Other possible sarcomatous differentiations include angiosarcoma, chondrosarcoma, and all other types of sarcomatous differentiations. Among them, liposarcomatous differentiation has been very rarely reported (4).

On mammography, most of the phyllodes tumors were found as a round shaped, circumscribed marginated, and high density mass, with large and fast-growing tendency.
A lucent halo caused by a fat component can localize the margin of the mass. Calcifications are rare because of their rapid growth (9). In our case, the mass showed typical features on mammography five years ago, with an oval circumscribed equal density mass with a radiolucent halo. The re-emerged mass appeared similar at first but evolved as an oval indistinct high-density mass, with heterogeneous internal fat components. The differences in these 

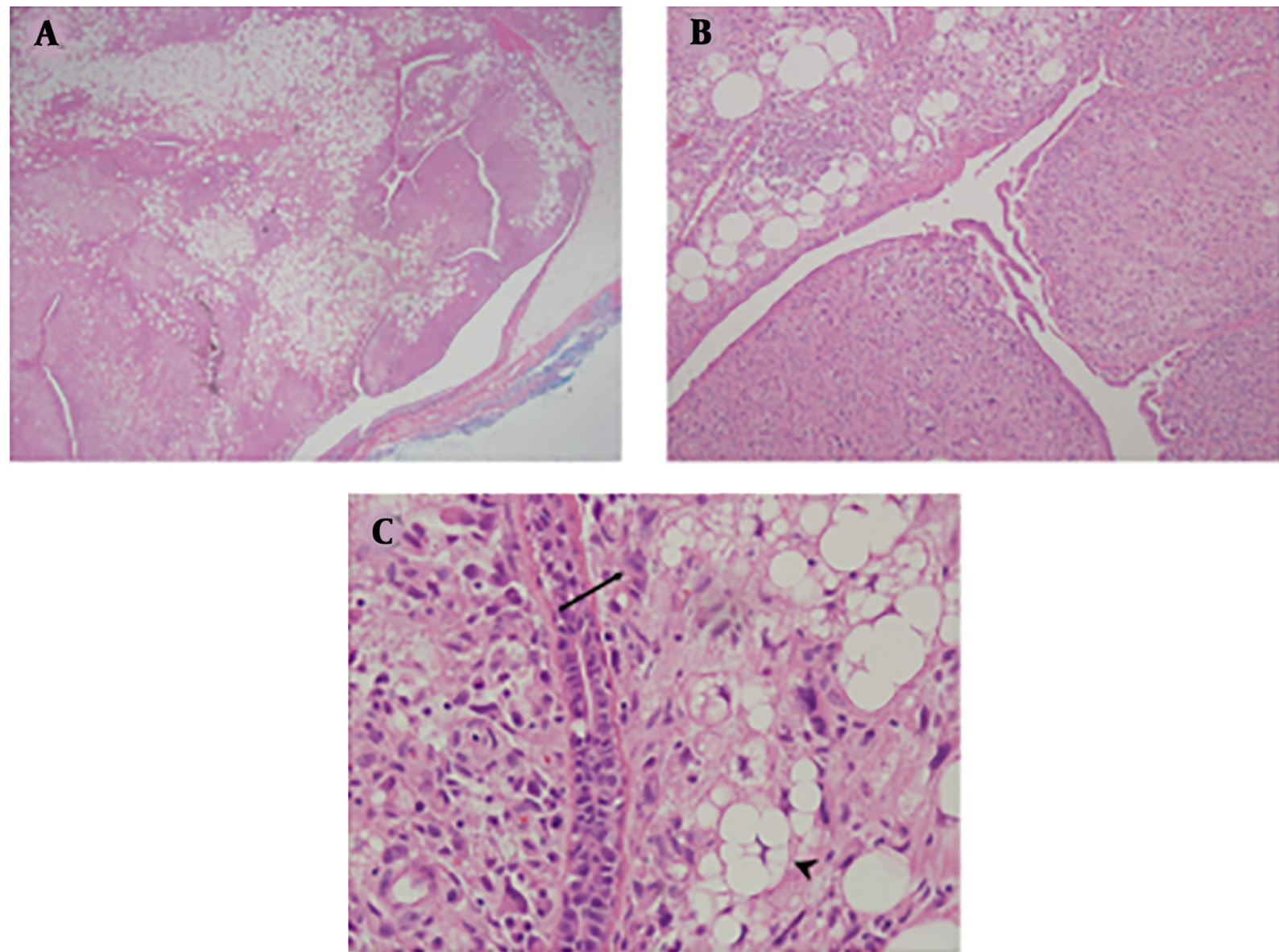

Figure 4. Microscopic findings of the tissue obtained after excision and biopsy. The tumor had a leaf-like growth pattern with marked stromal hypercellularity. (hematoxylin and $\operatorname{cosin}(\mathrm{H} \& \mathrm{E}) \times 40)(\mathrm{A})$. The epithelial component showed a benign-looking appearance, the stromal component composed of severe pleomorphic polygonal and spindle cells (arrow) with dispersed lipoblasts (arrowhead). (H\&E x100 and $\times 400)(B, C)$.

mammographic findings can be interpreted as the difference between malignant potentials.

On USG, most of the phyllodes tumors exhibited a lobulated contour, smooth margins, mild hypoechoic internal echo, and heterogeneous internal echo pattern (5). According to Liberman et al. most of phyllodes tumors exhibited hypoechoic internal echotexture and posterior acoustic enhancement and the presence of cystic spaces on USG was known to be more common in malignant cases (6). According to Kalambo et al. an irregular shape, noncircumscribed margins, and/or mass size $>7 \mathrm{~cm}$ may indicate more borderline and malignant subtypes of phyllodes tumors (7). In our case, the mass was observed as a mainly hyperechoic mass, with oval shape, indistinct margin, and cystic portions. It is noteworthy that the mass was hyperechogenic unlike usual cases, and the mass was with cystic portions, increasing the probability of malignancy.

Most phyllodes tumors are surgically treated and full mastectomy is recommended. If the mastectomy is not performed completely or if the resection margin is positive, recurrence may occur (10). The recurrence occurred in about $21 \%$ of all cases, most of which occurred within 2 years after surgery (1). In our case, recurrence may be suspected, because of the positive surgical margins seen in the previous surgery and the reappearance of the tumor near the previous operation site.

According to Chao et al. of the 110 cases, almost all phyllodes tumors appeared as hypoechoic, except for three hyperechoic lesions and one isoechoic lesion. Additionally, all four non-hypoechoic tumors were benign tumors (5). On the other hand, referring to case reports of malignant phyllodes tumors with heterologous sarcomatous differentiation, it was found that these tumors showed various echogenicity on USG, ranged from heterogeneous echogenicity to predominantly cystic mass $(11,12)$. In studying these case reports, we thought that the unique USG 
findings found in our case report were also related to heterogeneous sarcomatous differentiation. However, there were not many case reports showing hyperechoic appearance, and only one case was found. That case presented a patient with liposarcomatous differentiation showing hyperechoic on USG, same as our case (8). Considering these two cases together, although there is no published paper regarding the relationship between liposarcomatous differentiation and hyperechoic appearance, it can be interpreted in relation to the fact that fat is originally hyperechoic on USG.

Our case report included serial mammographies, USG images including Doppler USG, and microscope slides with pathologic explanations, compared to the previous case report, which only presented one USG image. However, since there were only two related case reports, there is a limit to the explanation of the association between liposarcomatous differentiation and hyperechoic appearance.

In conclusion, we can identify several potential rare features of various and unpredictable phyllodes tumors through our case report. First, malignant phyllodes tumors may appear as hyperechoic on USG, and second, they may show liposarcomatous differentiations, with these two factors possibly being related. In addition, phyllodes tumors can recur if they are not totally resected and they can show different malignant potentials from the previous tumor.

\section{Footnotes}

Authors' Contributions: Acquisition, analysis and interpretation of data was performed by Seung Min Kang. Drafting and critical revision of the manuscript was carried out by Seung Min Kang. Study concept, design, and supervision was done by Shin Young Kim. Administrative, technical, and material support was performed by Sun Huh, Si Hyong Jang, and Jong Eun Lee. The final version of the manuscript was approved by all authors.

Conflict of Interests: The authors declare that they have no conflict of interest.

Ethical Approval: This case report was approved by the Institutional Review Board. Informed consent was acquired from individual participant.

Financial Disclosure: None declared.

Funding/Support: This work was supported by the Soonchunhyang University Research Fund.

\section{References}

1. Lakhani SR, Ellis IO, Schnitt SJ. World Health Organization classification of tumours of the breast. Lyon, France: IARC; 2012. p. 143-7.

2. Tse GM, Niu Y, Shi HJ. Phyllodes tumor of the breast: An update. Breast Cancer. 2010;17(1):29-34. doi: 10.1007/s12282-009-0114-Z. [PubMed: 19434472].

3. Mangi AA, Smith BL, Gadd MA, Tanabe KK, Ott MJ, Souba WW. Surgical management of phyllodes tumors. Arch Surg. 1999;134(5):487-92. discussion 492-3. doi: 10.1001/archsurg.134.5.487. [PubMed: 10323420].

4. Powell CM, Rosen PP. Adipose differentiation in cystosarcoma phyllodes. A study of 14 cases. Am J Surg Pathol. 1994;18(7):720-7. doi: 10.1097/00000478-199407000-00008. [PubMed: 8017566].

5. Chao TC, Lo YF, Chen SC, Chen MF. Sonographic features of phyllodes tumors of the breast. Ultrasound Obstet Gynecol. 2002;20(1):64-71. doi: 10.1046/j.1469-0705.2002.00736.x. [PubMed: 12100421].

6. Liberman L, Bonaccio E, Hamele-Bena D, Abramson AF, Cohen MA, Dershaw DD. Benign and malignant phyllodes tumors: Mammographic and sonographic findings. Radiology.1996;198(1):121-4. doi: 10.1148/radiology.198.1.8539362. [PubMed: 8539362].

7. Kalambo M, Adrada BE, Adeyefa MM, Krishnamurthy S, Hess K, Carkaci $\mathrm{S}$, et al. Phyllodes tumor of the breast: Ultrasound-pathology correlation. AJR Am J Roentgenol.2018;210(4):W173-9. doi: 10.2214/AJR.17.18554. [PubMed: 29412020].

8. Zujić PV, Grebić D, Tomašić AM. Rare malignant phyllodes tumor with heterologous liposarcomatous differentiation. med Flum. 2016;52(2):253-6.

9. Tan $\mathrm{H}$, Zhang S, Liu H, Peng W, Li R, Gu Y, et al. Imaging findings in phyllodes tumors of the breast. Eur J Radiol. 2012;81(1):e62-9. doi: 10.1016/j.ejrad.2011.01.085. [PubMed: 21353414].

10. Reinfuss M, Mitus J, Duda K, Stelmach A, Rys J, Smolak K. The treatment and prognosis of patients with phyllodes tumor of the breast: an analysis of 170 cases. Cancer. 1996;77(5):910-6. doi: 10.1002/(sici)10970142(19960301)77:5<910::aid-cncr16>3.0.co;2-6. [PubMed: 8608483].

11. Murthy KP, Chakravarthy RP. Malignant phyllodes tumor with chondrosarcomatous differentiation: Radiological-pathological correlation. J Clin Imaging Sci. 2014;4:52. doi: 10.4103/2156-7514.141910. [PubMed: 25337438]. [PubMed Central: PMC4204296].

12. Warrier S, Hwang SY, Gibbings K, Carmalt H, O’Toole S. Phyllodes tumour with heterologous sarcomatous differentiation: Case series with literature review. Int I Surg Case Rep. 2015;11:91-4. doi: 10.1016/j.ijscr.2015.02.009. [PubMed: 25956039]. [PubMed Central: PMC4446672]. 